\title{
List of Reviewers 2013
}

Eastern Economic Journal (2014) 40, 284. doi:10.1057/eej.2014.11

The Editors and Editorial Board wish to acknowledge the assistance of the following colleagues, who reviewed articles during 2013, for their contributions of time and experience. Without their dedication, our work in publishing this journal could not go forward.

Benigno Aguirre
Abdullah Al-Bahrani
David Amdur
Aliaksandr Amialchuk
Lori Anderson
Laura Argys
Susan Averett
Sarah Baird
Cynthia Bansak
Jason Barr
Carlos Barros
Reagan Baughman
Jessica Bean
Bruce Blonigen
Rene Boheim
James Boyce
Kenneth Brown
James Butkiewicz
Maria Cancian
Charles Carlstrom
Kelly Carter
Yoo-Mi Chin
Lesley Chiou
Shin-Yi Chou
Catherine Clau
David Colander
Gregory Colman
Steven Cook
Hope Corman
Mark Crain
Dhaval Dave
Lewis Davis
Christopher Decker
Marina Della Giusta
James DeVault
Beatrice D’Hombres
Angela Dills
Mathias Dolls
Paul Dourgnon
Nadia Doytch

Ryan Edwards
Bruce Elmslie
Sarah Estelle
Maoyong Fan
Beatrice Farkas
Dietmar Fehr
Nicholas Feltovich
Sherman Folland
Wan-Ju Iris Franz
Bernd Frick
Gregory Givens
Irina Grafova
Matthew Gregg
Amanda Griffith
George Hall
Timothy Halliday
Kyle Hampton
John Harter
Frank Heiland
Rey Hernandez-Julian
Randall Holcombe
Julie Hotchkiss
Brad Humphreys
Maria Iacovou
Eric Isenberg
Tor Iverson
Joyce Jacobsen
Stephen Jenkins
Helen Jensen
Jaewon Jung
Florence Jusot
Michael Kelly
Hashmat Khan
Anthony
Krautmann
Jonathan Lafky
Richard Langlois
Audrey Laporte
Sarah Larson
Marc Lavoie

Dong Jin Lee
Michael Leeds
Lei Li
Fernando Lozano
Zijun Luo
Melissa Mahoney
Liam Malloy
Daniel Marburger
Brendan Markey-Towler
Nikolay Markov
Jaime Marquez
Victor Matheson
Fabio Mattos
Kerry Anne McGeary
Caitlin Knowles Meyers
Brian Mills
Javier Miranda
Donka Mirtcheva
Kusum Mundra
George Naufal
Erik Nesson
Kelly Noonan
Tomson Ogwang
Orgul Ozturk
Yong Jin Park
Rodney Paul
Anita Pena
Andrew Pickering
Claus Portner
Andrea Presbitero
Joseph Price
Nancy Reichman
Luc Renneboog
Lorenzo Rocco
Philip Rothman
Christopher Ruebeck
Daniel Scheer
Bradley Schiller
Jonathan Schwabish
Julia Schwenkenberg

\author{
Bruce Seaman \\ M. Fuat Sener \\ Cristian Sepulveda \\ Mark Setterfield \\ Elias Shukralla \\ Asia Sikora \\ Nicholas Sim \\ Curtis Simon \\ Nicolas Sirven \\ Mikal Skuterud \\ John Solow \\ Chad Sparber \\ Patrick Stephan \\ Max Stephenson, Jr. \\ Courtenay Stone \\ Caleb Stroup \\ Sarinda Taengnoi \\ Fangfang Tan \\ Hiroshi Tanaka \\ Shaun Tanger \\ Sabrina Terrizzi \\ M. Kathleen \\ Thomas \\ Kevin Tsui \\ Craig Upright \\ Victor Valcarcel \\ Michele Ver Ploeg \\ Peter von Allman \\ James Walker \\ Miao Wang \\ X. Henry Wang \\ Yang Wang \\ Robert Weathers \\ Charles Weise \\ Jeffrey Wenger \\ Yan Wu \\ Muzhe Yang \\ Kevin Zhang \\ Martin Ziegelbaeck \\ Thomas Zylkin
}

